Gut, 1989, 30, 1731-1736

\title{
Comparative DNA analyses in longstanding ulcerative colitis with aneuploidy
}

\author{
R LÖFBERG, T CASPERSSON, B TRIBUKAIT, Å ÖST
}

From the Department of Medicine, Huddinge University Hospital, Department of Medical Radiobiology and Pathology, Karolinska Hospital and Institute, Stockholm, Sweden

SUMmary In seven patients with longstanding ulcerative colitis, multiple mucosal biopsies taken at colonoscopy were assessed for DNA content and histological dysplasia. DNA analyses were performed using flow cytometric analyses in biopsies and microspectrophotometry in imprint slides prepared from the biopsies. Abnormal aneuploid DNA content was detected in five of the patients in eight separate locations in the colon. There was good conformity between the two methods in the detection of aneuploidy. Both methods of DNA analysis are considered to be applicable in mucosal biopsies from patients with ulcerative colitis. In two patients with significant low grade dysplasia in association with macroscopical lesions (DALMs), there was a relationship with aneuploid DNA pattern found by flow cytometric or microspectrophotometric analyses. Indefinite changes, probably dysplastic were found in three other patients and two of those also displayed aneuploidy, although only once in the same biopsy location. In five locations aneuploidy was detected without concomitant dysplasia. DNA-aneuploidy seems therefore to appear earlier than dysplasia and may be an early marker of malignant transformation of the mucosa in ulcerative colitis. Prospective trials are needed before the clinical significance of early findings of aneuploidy can be determined.

Abnormal, aneuploid DNA pattern has been shown in $54-85 \%$ of colorectal carcinomas. ${ }^{1-4}$ Aneuploidy has also been described in mucosal biopsies from premalignant conditions in the colon such as familial polyposis, adenomas and longstanding ulcerative colitis. $^{-i}$ We have previously reported a close relationship between DNA aneuploidy and dysplasia/ carcinoma in ulcerative colitis in a prospective, endoscopical study" and similar results have been found in a large retrospective study on colectomy specimens from patients with colitis carcinoma and dysplasia."

In these studies, DNA analyses have been conducted utilising the rapid flow cytometric technique. With flow cytometric analysis the DNA pattern in a suspension of a large number of cells (several thousand) can be determined in a short period of time, making this method suitable for routine clinical

Address for correspondence: Dr Robert lotherg. (iastrocenterology Unu. Medical Dept. Huddinge University Hospital, S-141 86 Huddinge. Sweden. Accepted for publication 31 March 1989. work. Using the more time consuming microspectrophotometric method in slide preparations, the DNA content in single selected cells can be measured. The latter method has been extensively used in DNA studies of various malignancies, and has also been shown to correlate well with flow cytometric analysis."'" A retrospective microspectrophotometry study of resected colorectal specimens from ulcerative colitis patients with carcinoma or dysplasia revealed a progression of DNA abnormality as the severity of the dysplastic lesions increased.'?

In order to compare the microspectrophotometry analysis with the flow cytometric method in patients with longstanding ulcerative colitis a prospective study was carried out. The main goal was to evaluate if microspectrophotometry analysis could be applied to imprint slides of biopsies from colonic mucosa, and to correlate abnormal DNA pattern found by using this technique with aneuploidy found at flow cytometric analysis. Furthermore, the aim was to correlate findings of histological dysplasia with the result from the DNA analyses. 


\section{Methods}

PATIENTS

From an established surveillance programme for ulcerative colitis patients of long duration, ${ }^{13}$ seven cases were selected for this study. All patients had total, quiescent colitis with a median duration of 24 years (range 11-37). In four of the patients aneuploidy at flow cytometric analysis had previously been detected at colonoscopy and in three of these dysplasia had been present. Isolated dysplasia had been found in one additional patient. Two patients without preceding dysplasia or ancuploidy were included for control purposes.

COLONOSCOPY AND BIOPSY SAMPLING

Total colonoscopy (Olympus CF1T10L) was undertaken in all patients except one, where flexible sigmoidoscopy was carried out. Biopsies were sampled from 10 predetermined locations in the colon (1 caecum, 2 ascending colon, 3 hepatic flexure, 4 proximal and 5 distal transverse, 6 splenic flexure, 7 proximal and 8 distal descending colon, 9 sigmoid and 10 rectum). If necessary fluoroscopy was used to locate the anatomical position of the tip of the instrument. In each location two biopsies were taken for histopathological assessment, and one biopsy taken immediately adjacent (0-2 $\mathrm{mm}$ apart) was taken for flow cytometric analysis. In patients where aneuploidy had been detected previously the flow cytometric biopsies from each location were analysed separately, otherwise they were pooled into three fractions $(1-3,4-6$, and 7-10). Imprint slides for microspectrophotometry DNA analysis were instantly prepared using the biopsy specimens in each location if the patient had been found to have ancuploidy before, or from location 2, 5, and 8 if aneuploidy had not previously been found. Additional biopsies were taken if macroscopical lesions were detected.

FLOW CYTOMETRIC DNA ANALYSIS

Biopsies for flow cytometric DNA analysis were placed in saline, pressed through a nylon grid (40-50 mesh counts $/ \mathrm{cm}$ ) and the resulting cell suspension fixed in ice cold ethanol. The cells were then treated with an isotonic Tris EDTA buffer (Tris $0 \cdot 1 \mathrm{M}, \mathrm{NaCl}$ $0.07 \mathrm{M}$ and EDTA $0.005 \mathrm{M}, \mathrm{pH} 7.5)$ containing RNAse $(1 \mathrm{mg} / \mathrm{ml})$ in order to eliminate the RNA. After pepsin treatment $(0.5 \%$ pepsin solution, $\mathrm{pH}$ $2 \cdot 0$ ) for 10 minutes in a $37^{\circ} \mathrm{C}$ water bath, suspensions of single cell nuclei were obtained. The DNA was stained with $2.5 \times 10^{-5} \mathrm{M}$ ethidium bromide in Tris EDTA buffer with a molarity of $395 \mathrm{mOsmol}$.

DNA content was analysed using a rapid flow cytofluorometer (ICP 11) with a flow rate of up to
1000 cells/sec. The excitation and emission wavelengths were $455-490 \mathrm{~nm}$ and $590-630 \mathrm{~nm}$ respectively. The output was sorted by a 256 channel analyser and was presented as a histogram. The DNA values of the analysed cells were calculated in relation to normal diploid human lymphocytes in order to get a diploid reference point. The ploidy level of the cell population was defined as the DNA value of the $G_{1} / G_{0}$ cells in relation to the ploidy level of normal diploid cells (2c). The coefficient of variation was less than $3 \%$ it.

Cell suspensions with a $G_{1} / G_{0}$-peak at $2 \cdot()$ c and a corresponding $\mathrm{G}_{2}+\mathrm{M}$ peak at $4 \cdot 0 \mathrm{c}$ deviating less than $10 \%$ from the standard lymphocytes were considered to be diploid. Additional distinct $\mathrm{G}_{1} / \mathrm{G}_{0^{-}}$ peak(s) with c-values exceeding $2 \cdot 2 \mathrm{c}$ were considered as aneuploid. Peaks in the tetraploid region of $4 \cdot 0 \mathrm{c}$ exceeding the normal $\mathrm{G}_{2}+\mathrm{M}$ peak more than 3 SD were classified as ancuploid if also a corresponding peak at $8 \cdot 0 \mathrm{c}$ was identified.

MICROSPECTROPHOTOMETRY DNA ANALYSIS

The imprint slides were air dried and fixed in $4 \%$ formaldehyde for a minimum of 12 hours, then rinsed in distilled water and stained by the Feulgen method. ${ }^{15}$ Acid hydrolysis in $5 \mathrm{M} \mathrm{HCl}$ at $22^{\circ} \mathrm{C}$ for 60 minutes was followed by application of Schiff reagent for two hours in the dark at room temperature. The preparation was rinsed in distilled water and sulphite.

The prepared slides were examined by light microscopy and were found to contain scattered clusters of epithelial cells as well as dispersed, free lying epithelial cells. These were easy to separate from fibroblasts or lymphocytes. During the identification process a selection was made in favour of larger or darker cell nuclei as these could be supposed to have abnormal DNA content. Only intact cell nuclei were selected using $\times 100$ magnification, and the DNA content of each nucleus was determined by extinction measurements in monochromatic light at 546 $\mathrm{nm}$, using a rapid scanning and integrating microspectrocytophotometer developed by Caspersson. ${ }^{1017}$ The extinction

$$
\left(=\log \frac{100 \%}{\text { transmission \% }}\right)
$$

is a linear function of the DNA-amount and was measured at $500-10000 \cdot 16 \mu \mathrm{m}^{2}$ large spots covering each nucleus. The total DNA amount for each cell was computed and registered on a printer.

An average of 100 cells were systematically scanned and measured in each slide. Segmented granulocytes were measured as internal, diploid control cells. The result was presented as a histogram. DNA histograms from the microspectrophotometry analyses were classified as diploid with one 

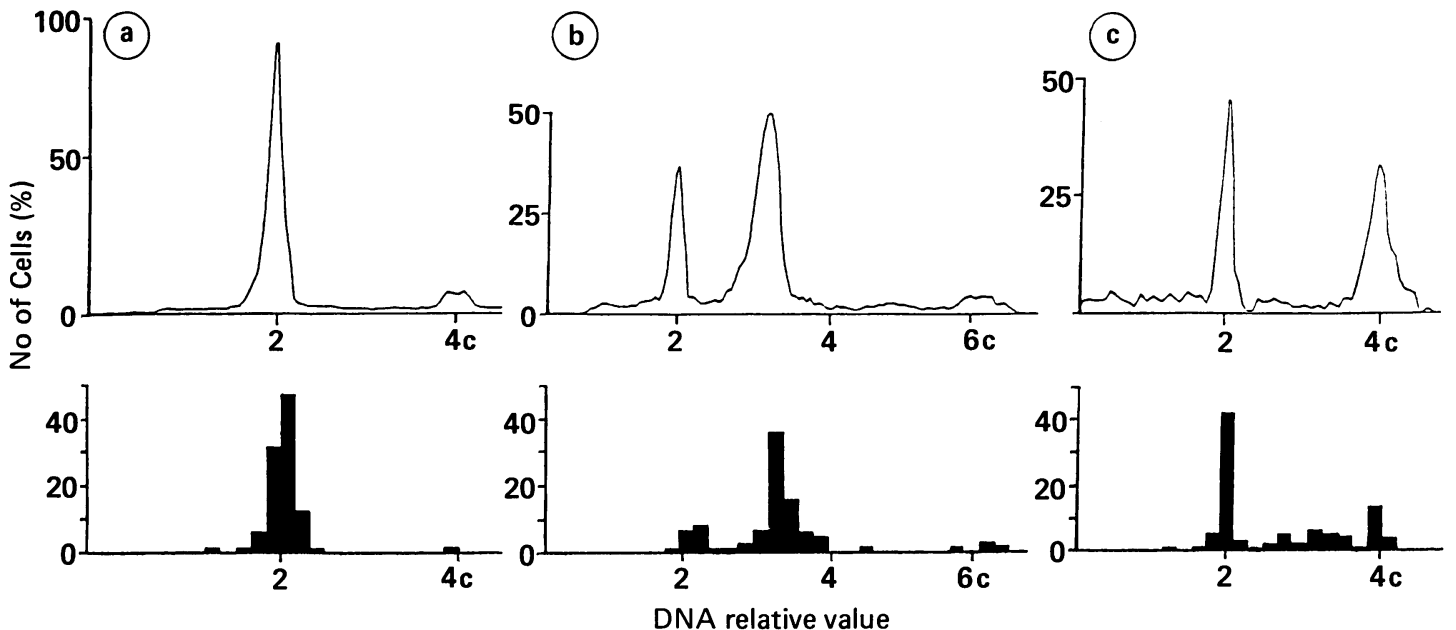

Fig. 1 (a) Normal, diploid DNA histograms with flow cytometric (FCM) analysis (top) and microspectrophotometry (MSP) analysis (bottom) displaying a single, distinct peak at $2 c$. (b) Aneuploid peak at $3 \cdot 3$ c and a corresponding $G_{2}+M$ peak above $6 \mathrm{c}$ in the flow cytometric as well as the microspectrophotometry histogram. (c) Aneuploidy at $4 \cdot 0 \mathrm{c}$ is shown by both analyses and a scattered pattern between 2 and $4 c$ is also seen in the microspectrophotometry histogram.

distinct peak in the $2 \mathrm{c}$ region, or aneuploid if distinct extra peaks could be identified or a scattered pattern with DNA values exceeding $4 \mathrm{c}$ was found (modified from $\left.^{18}\right)$. In Figure 1a a diploid microspectrophotometry histogram is shown together with the corresponding, diploid flow cytometric analysis.

\section{HISTOPATHOLOGICAL ASSESSMENT}

Grading of dysplasia findings was made in line with previous reports according to the classification adopted by Riddell et al. ${ }^{19}$ Assessment was carried out in a blinded manner without knowledge of the results of neither flow cytometric nor microspectrophotometry DNA analyses. Changes interpreted as (1) indefinite, probably dysplastic, and definite dysplasia of (2) low, or (3) high grade were recorded.

\section{Results}

A total of 43 samples were analysed by both techniques. In one sample the imprint preparation was technically default and no microspectrophotometry analysis could be performed. All flow cytometric analyses were technically acceptable with interpretable histograms. In all four patients where aneuploidy had previously been detected by flow cytometric analyses, this finding was reproduced, albeit not always in exactly identical locations in the colon as before. Aneuploidy at flow cytometric analyses was detected in seven biopsies from these patients (Table). The corresponding microspectrophotometry analyses on imprint slides from these biopsies all showed aneuploidy and in six of these, the microspectrophotometry histograms had distinct aneuploid peaks enabling their c-values to be calculated (Fig. 1b, c). The seventh microspectrophotometry histogram had a scattered aneuploid pattern above $4 \mathrm{c}$ without a distinct peak. The corresponding flow cytometric analysis showed an aneuploid peak at 3.8c. In one further imprint sample, scattered aneuploidy was detected with microspectrophotometry, but the corresponding, pooled flow cyto-

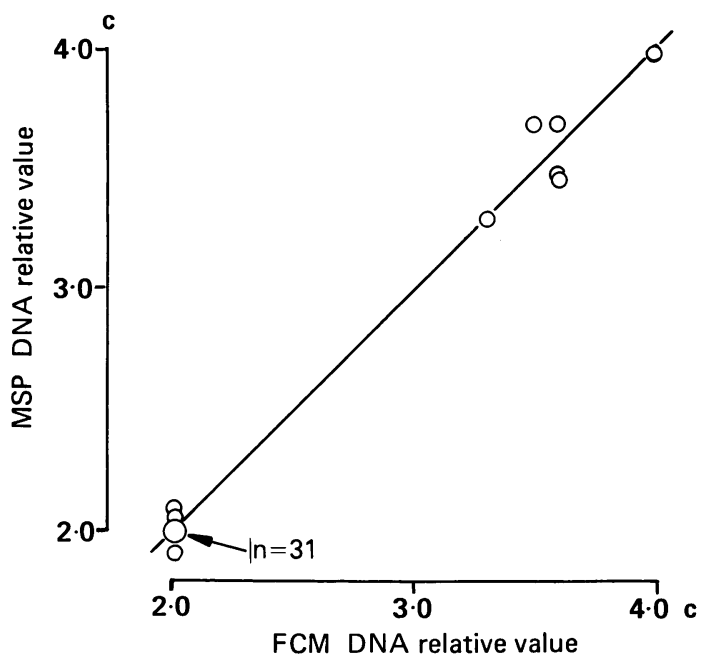

Fig. 2 Relationship between diploid peaks in 34 samples and DNA-aneuploid peak values in six samples as analysed by flow cytometry (FCM) in biopsies and microspectrophotometry $(M S P)$ in imprint slides $(y=-0.01+1.01 x, r=0.997)$. 
Table Results of flow cytometric and microspectrophotometry DNA analyses from biopsy specimens at 10 predetermined locations in the colon and rectum in seven patients with longstanding ulcerative colitis compared with histological findings of dysplasia

\begin{tabular}{|c|c|c|c|c|c|c|c|c|c|c|c|}
\hline $\begin{array}{l}\text { Patient } \\
\text { no }\end{array}$ & Analyses & $\begin{array}{l}\text { Locations } \\
I\end{array}$ & 2 & 3 & 4 & 5 & 6 & 7 & 8 & 9 & 10 \\
\hline \multirow[t]{3}{*}{1} & $\mathrm{FCM}$ & () & $\mathrm{An} / 4 \cdot 0$ & () & 0 & 0 & 0 & () & 0 & 0 & 0 \\
\hline & MSP & () & $\mathrm{An} / 4 \cdot 0$ & () & 0 & () & 0 & 0 & 0 & () & () \\
\hline & Dysplasia & () & 0 & () & 1 & 0 & 0 & () & 0 & () & 0 \\
\hline \multirow[t]{3}{*}{2} & FCM & () & () & 0 & 0 & () & 0 & () & 0 & $\mathrm{An} / 3 \cdot 6$ & 0 \\
\hline & MSP & () & () & () & - & () & 0 & 0 & 0 & $\mathrm{An} / 3.5$ & () \\
\hline & Dysplasia & () & () & () & () & 0 & () & () & 0 & () & () \\
\hline \multirow[t]{3}{*}{3} & FCM & () & () & 0 & () & $\mathrm{An} / 3 \cdot 6$ & 0 & $\mathrm{An} / 3.5$ & $\mathrm{An} / 3 \cdot 8$ & () & 0 \\
\hline & MSP & () & () & 0 & 0 & $\mathrm{An} / 3.5$ & 0 & $\mathrm{An} / 3.7$ & $\mathrm{An} / \mathrm{sc}$ & () & () \\
\hline & Dysplasia & () & () & () & 0 & 1 & () & () & 0 & () & 0 \\
\hline \multirow[t]{3}{*}{4} & FCM & - & - & - & - & - & - & () & 0 & $\mathrm{An} / 3 \cdot 3$ & $\mathrm{An} / 3 \cdot 6$ \\
\hline & MSP & - & - & - & - & - & - & 0 & 0 & $\mathrm{An} / 3 \cdot 3$ & $\mathrm{An} / 3.7$ \\
\hline & Dysplasia & - & - & - & - & - & - & () & 0 & 2/DALM & () \\
\hline \multirow[t]{3}{*}{5} & $\mathrm{FCM}$ & -1 & - & () & - & $-1)$ & - & - & 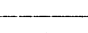 & 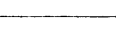 & - - \\
\hline & MSP & - & - & () & - & () & - & - & $\mathrm{An} / \mathrm{sc}$ & - & - \\
\hline & Dysplasia & () & 1 & 2/DALM & 1 & 2 & 1 & 0 & 1 & 1 & 1 \\
\hline \multirow[t]{3}{*}{6} & FCM & - & -1) & & + & - & 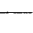 & - & & - & \\
\hline & MSP & - & () & - & - & 0 & - & - & () & - & - \\
\hline & Dysplasia & () & () & () & () & () & 1 & () & () & 1 & () \\
\hline \multirow[t]{3}{*}{7} & FCM & 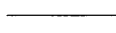 & -1) & - & 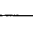 & $-1)$ & & 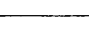 & $\ldots$ & 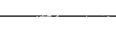 & \\
\hline & MSP & - & () & - & - & 0 & - & - & 0 & - & - \\
\hline & Dysplasia & 0 & () & 0 & 0 & 0 & 0 & () & 0 & () & 0 \\
\hline
\end{tabular}

Locations in the colon: $1=$ caecum, $2=$ ascending, $3=$ hepatic flexure, $4=$ proximal transverse, $5=$ distal transierse, $6=$ splenic flexure, $7=$ proximal descending, $8=$ distal descending, $9=$ sigmoid, $10=$ rectum.

DNA analyses: $F C M=$ flow cytometric,$M S P=$ microspectrophotometry, ()$=$ diploid. An= aneuploidy/relative $c-v$ alue of aneuploid peak, sc= scattered aneuploidy $>4 \cdot 0 \mathrm{c}$ in MSP-analysis. $\longrightarrow-10$ pooled FCM-samples. $-=$ no analysis performed.

Dysplasia: $0=$ negative, $1=$ indefinite changes, probably dysplastic, $2=$ low grade, DALM=dysplasia-associated lesion or mass.

metric analysis were diploid. Figure $1 \mathrm{~b}$ and $\mathrm{c}$ illustrates analyses from two separate biopsies with aneuploidy. The relationship between the relative c-values of the normal, diploid histograms and the six analyses showing distinct aneuploid peaks as measured by flow cytometric and microspectrophotometry technique respectively is visualised in Figure 2. The correlation was established with $\mathrm{r}=0.997$.

Dysplasia, altogether found in 13 biopsies, was mainly of the indefinite, probably dysplastic grade. Only three biopsies showed dysplasia of low grade. No high grade dysplasia or carcinoma was detected. One patient (no 4) with low grade dysplasia in combination with a macroscopical lesion (so called 'dysplasia-associated lesion or mass' $=$ DALM) also had aneuploidy in the same lesion as analysed by flow cytometric and microspectrophotometry technique. One further patient (no 5) had a DALM with low grade dysplasia in the hepatic flexure as well as dysplasia in flat mucosa elsewhere in the colon. Flow cytometric analysis revealed no aneuploidy but the microspectrophotometry histogram representing the distal colon (location 8) was clearly aneuploid with a scattered DNA pattern. In this location changes classified as indefinite, probably dysplastic, were detected. Patient no 3 with aneuploidy in three different locations $(5,7$, and 8$)$ had indefinite changes, probably dysplasia in only one of these locations. One further patient had aneuploidy in one location and indefinite changes, probably dysplastic in another. The two control patients were normal concerning DNA-content, but one patient had indefinite changes probably dysplastic in two locations.

\section{Discussion}

In this study abnormal, ancuploid DNA pattern in mucosal biopsies from patients with longstanding ulcerative colitis was shown with flow cytometric technique as well as with microspectrophotometric analyses. There was a good conformity between the two methods used, both in terms of detection of aneuploidy in the colon as well as ploidy level. The latter is in agreement with other reports comparing both methods in established malignancies - for example, mammary carcinoma"' and sarcoma."

When using the flow cytometric method a large number of cells are rapidly assessed concerning the DNA content and a DNA profile of the whole biopsy specimen is obtained. When dealing with nonmalignant tissue, the majority of cells analysed will have a normal (diploid) DNA content. These cells form the diploid background from which 
an aneuploid cell population, if large enough, will emerge as a separate peak(s). When several thousand normal cells are analysed it is obvious that small amounts of definitely aneuploid cells may drown in this background, even though the flow cytometric technique is attributed with high resolution and low coefficient of variation. This may explain why aneuploidy could not be shown by flow cytometric analyses in patient no 5 as the four biopsies from the left colon and rectum were pooled together. Nevertheless, we were able to show aneuploidy in pooled biopsies in our previous study, but this could be explained by larger aneuploid cell populations.

Single cell DNA measurements done with the microspectrophotometry method make it possible to identify small numbers of cells with an abnormal DNA content. In this respect the microspectrophotometry method may be more sensitive in the detection of early, limited aneuploid cell populations in an otherwise normal mucosa. In this study such a situation was noted in one case only (no 5). Interestingly, there was a strong correlation between findings of aneuploidy by flow cytometric analyses and the corresponding microspectrophotometry analyses, even though the imprint slides for the latter only represent cells from the outer layers of the biopsy specimen. It appears as if either method could be applicable when mucosal biopsies in longstanding ulcerative colitis are analysed.

The findings of dysplasia in this study was sparse although two patients did display macroscopical lesions with low grade dysplasia, and aneuploidy was detected in both cases. Low grade dysplasia in association with macroscopical lesion is normally considered to be an indication for surgical intervention with proctocolectomy in cancer surveillance programmes for ulcerative colitis,,$^{21}$ and hence of clinical significance. In one case aneuploidy was detected within the lesion and in the other aneuploidy was found elsewhere in the colon. In this respect the correlation with clinically significant dysplasia was good. The clinical significance of indefinite changes, probably dysplastic, in ulcerative colitis is, however, unclear; sometimes they precede development of more severe dysplasia and sometimes they may disappear. ${ }^{13}$

In two of the five patients with DNA aneuploidy this was detected in more than one location in the colorectum, indicating that the mucosa was more extensively affected. This was also the case in four of five patients with longstanding ulcerative colitis with DNA aneuploidy in our previous, prospective study. ${ }^{x}$ We then also noted that two patients displayed DNA aneuploidy without concomitant dysplasia, and this was also seen in another prospective study from
Sweden. ${ }^{21}$ Similar observations have also been made in a retrospective study of colectomy specimens from patients with ulcerative colitis and carcinoma of the colorectum" using flow cytometric analyses. In that study, DNA aneuploidy was found distant from the carcinoma in $32 \%$ of the patients and in $9 \%$ also in non-dysplastic mucosa. Retrospective microspectrophotometry analyses ${ }^{12}$ have also revealed aneuploid DNA pattern in non-cancerous/non-dysplastic mucosa in ulcerative colitis specimens. It is noteworthy that patients with idiopathic colorectal carcinoma rarely have DNA aneuploidy in the mucosa 2-10 cm distant from the tumour.' As aneuploidy is closely related to malignancy, findings of aneuploidy in a yet non-dysplastic mucosa in ulcerative colitis as found in this and our previous study, ${ }^{\circ}$ as well as in others, ${ }^{74}{ }_{12} 21$ could be considered as a sign of malignant transformation, and may consequently also be an early warning. The lead time before the development of morphologically detectable malignancy is still unknown, but in this respect the two previously reported patients, who both developed carcinoma within one year following the findings of aneuploidy, are of interest. ${ }^{x=}$ Unfortunately, neither of those patients had a preceding dysplasia or aneuploidy negative colonoscopy. One patient in our previous study ${ }^{*}$ has had persistent DNA aneuploidy without dysplasia for six years, and a case with persistent aneuploidy for seven years, with only low grade dysplasia once and no development of carcinoma, has been reported. ${ }^{23}$ This may indicate that, at least in some patients with ulcerative colitis, the time interval between the occurrence of DNA aneuploidy and morphologically manifest malignancy in the colon is substantial. This could have important clinical impact on longterm surveillance in ulcerative colitis.

The only way to establish the clinical significance of findings of DNA aneuploidy in ulcerative colitis is by prospective trials. This presents an inherent ethical problem in the longterm follow up of ulcerative colitis patients, as the main interest in cancer surveillance programmes is to avoid carcinoma development. Thus the majority of potential carcinoma patients are operated on in an earlier stage due to findings of dysplasia. Hence, longterm follow up of aneuploidy in patients with longstanding ulcerative colitis most probably will have to end when significant dysplasia develops and the patient is recommended for surgery. The most appropriate way to further evaluate the use of flow cytometric or microspectrophotometry DNA analyses in order to detect aneuploidy in ulcerative colitis is to include the methods in a longterm, prospective surveillance programme.

A purely diploid or near diploid colorectal 
carcinoma may develop in around $20 \%$ of ulcerative colitis carcinoma patients as well as in idiopathic colonic carcinoma'" and in those cases, DNA analyses are not suitable for early detection as they are likely to reveal only gross chromosomal aberrations. A diploid DNA analysis is thus not a guarantee against carcinoma development.

The main advantage with the flow cytometric technique is that it allows rapid screening of a large number of samples. Provided that the fresh biopsy specimens are handled adequately and without delay to avoid disintegration of the cells before preparation. interpretation of the histogram presents no difficulties. Analyses with aneuploid peaks are easily recognised.

Preparation of single cell imprint slides require care, but when air dried, the slides are not sensitive to transport time. The benefit is the possibility to detect small amounts of ancuploid cells. The main obstacle when using the microspectrophotometry technique is the amount of time needed for each analysis. One single imprint slide of colonic mucosal cells requires two to four hours to examine. Ongoing development to create instruments with computerised automation will substantially increase the speed of the microspectrophotometry analyses and probably make this technique more applicable for routine clinical work.

This study was supported by grants from the Swedish Cancer Society, the Cancer Society of Stockholm and King Gustaf the V's Jubilee foundation.

\section{References}

1 Tribukait B, Hammarberg C, Rubio C. Ploidy and proliferation patterns in colorectal adenocarcinoma as related to Dukes' classification and histopathological differentiation. Acta Pathol Microbiol Immunol Scand Sect A 1983; 91: 89-95.

2 Armitage NC. Robins RA, Evans DF, Turner DR. Baldwin RW, Hardcastle JD. The influence of tumour cell DNA abnormalities on survival in colorectal cancer. Br J Surg 1985; 72: 828-30.

3 Kokal W. Sheibani K. Terz J, Harada JR. Tumor DNA content in the prognosis of colorectal carcinoma. JAMA 1986; 255: 3123-7.

4 Melamed MR, Euker W. Banner P, Janov AJ, Kessler G, Darzynkiewicz Z. Flow cytometry of colorectal carcinoma with three-year follow up. Dis Colon Rectum 1986; 29: 184-6.

5 Quirke P. Dixon MF, Day DW, Fozard JBJ, Talbot IC, Bird CC. DNA ancuploidy and cell proliferation in familial adenomatous polyposis. Gut 1988; 29: 603-7.

6 Goh HS, Jass JR. DNA content and the adenomacarcinoma sequence in the colorectum. J Clin Pathol 1986: 39: 387-92.

7 Tribukait B, Hammarberg C. DNA flow cytometry of endoscopic biopsies for detection of malignancy in ulcerative colitis. A study based on the DNA-patterns of gastrointestinal tumours. Acta Endoscopica 1985: 15: $257-63$

8 Löfberg R. Tribukait B. Öst Å. Brostöm O, Reichard H. Flow cytometric DNA analysis in longstanding ulcerative colitis: a method of prediction of dysplasia and carcinoma development? (Gut 1987; 28: $110($-6.

9 Melville DM, Jass JR. Shepherd NA, et al. Dysplasia and deoxyribonucleic acid in the assessment of precancerous changes in chronic ulcerative colitis. Gastroenterology 1988; 95: 668-75.

10 Auer G, Tribukait B. Comparative single cell and flow DNA analysis in aspiration biopsies from breast carcinomas. Acta Pathol Microbiol Scand Sect A 1980; 88: $35.5-8$.

11 Bauer HCF, Kreichergs A. Tribukait B. DNA microspectrophotometry of bone sarcomas in tissue sections as compared to imprint and flow DNA analysis. Cytometry 1986; 7: 544-50.

12 Cuvelier CA. Morson BC. Roels HJ. The DNA content in cancer and dysplasia in chronic ulcerative colitis. Histopathology 1987; 11: 927-39.

13 Broström O. Löfberg R. Öst $\AA$. Reichard H. Cancer surveillance of patients with longstanding ulcerative colitis - a clinical, endoscopical and histological study. Gut 1986: 27: 1408-13.

14 Tribukait B. Flow cytometry in surgical pathology and cytology of tumours of the genito-urinary tract. Adv Clin Cytol 1984: 2: 163-89.

15 Gaub J, Auer G, Zetterberg A. Quantitative cytochemical aspects of a combined Feulgen napthol yellow $S$ staining procedure for the simultaneous determination of nuclear and cytoplasmic protein and DNA in mamallian cells. Exp Cell Res 1975; 92: 323-32.

16 Caspersson T. Kudynowski J. Cytochemical instrumentation for pathological work. Int Rev Exp Pathol 1980): 21: $1-54$.

17 Caspersson T. Quantitative tumor cytochemistry GHA Clowes Memorial Lecture. Cancer Res 1979: 39: 2341-55.

18 Auer G, Caspersson T, Wallgren A. DNA content and survival in mammary carcinoma. Anal Quant Cytol 1980; 2: 161-5.

19 Riddell RH, Goldman H, Ransohoff D, et al. Dysplasia in inflammatory bowel disease: Standardized classification with provisional clinical applications. Hum Pathol 1983: 14: 931-66.

20) Lennard-Jones JE. Compliance, cost and common sense limit cancer control in colitis. Gut 1986; 27: 14(1)3-7.

21 Rutegărd J, Ähsgren L. Stenling R, Roos G. DNA content in ulcerative colitis: flow cytometric analysis in a patient series from a defined area. Dis Colon Rectum 1988: 31: 710-5.

22 Hammarberg C. Rubio C. Slezak P. Tribukait B. Öhman U. Flow cytometric DNA-analysis as a means for carly detection of malignancy in patients with chronic ulcerative colitis. Gut 1984: 25: 905-8.

23 Rutegărd J, Ǎhsgren L, Stenling R, Roos G. DNA content and mucosal dysplasia in ulcerative colitis: flow cytometric analysis in patients with dysplastic or indefinite morphologic changes in the colorectal mucosa. Dis Colon Rectum 1989. (In press). 